\title{
Ontological Boundaries Between Humans and Computers and the Implications for Human-Machine Communication
}

\author{
Andrea L. Guzman ${ }^{1}$ (D) \\ 1 Department of Communication, Northern Illinois University, DeKalb, Illinois, United States of America
}

\begin{abstract}
In human-machine communication, people interact with a communication partner that is of a different ontological nature from themselves. This study examines how people conceptualize ontological differences between humans and computers and the implications of these differences for human-machine communication. Findings based on data from qualitative interviews with 73 U.S. adults regarding disembodied artificial intelligence (Al) technologies (voice-based Al assistants, automated-writing software) show that people differentiate between humans and computers based on origin of being, degree of autonomy, status as tool/tool-user, level of intelligence, emotional capabilities, and inherent flaws. In addition, these ontological boundaries are becoming increasingly blurred as technologies emulate more human-like qualities, such as emotion. This study also demonstrates how people's conceptualizations of the human-computer divide inform aspects of their interactions with communicative technologies.
\end{abstract}

Keywords: human-machine communication, ontology, artificial intelligence, computers, humanity, mobile voice assistants, automated journalism, human-computer interaction, human-robot interaction

\section{Introduction}

Within the past two decades, technology companies have introduced numerous forms of communicative technologies, programs, and devices that function in the role of a communicator by exchanging messages with people or by performing a communicative task on their behalf (e.g., chatbots, automated-writing software). People now are interacting with communicators that are of a different ontological nature from themselves, prompting scholars to ask how ontological divides between communicators shape people's interactions 
with technology (e.g., A. P. Edwards, 2018). The goal of this study is to better understand aspects of the ontological dimensions of human-machine communication. To do so, the study focuses on two research questions: (1) How do people conceptualize the ontological differences between humans and computers? and (2) What are the implications of these ontological differences for the human-machine communication process?

This study contributes to the growing body of knowledge regarding the role of ontology in Human-Machine Communication (HMC), an emerging area of communication research focused on meaning-making between humans and machines (Guzman, 2018). In human communication, a person's behavior is guided by their interpretations of their communication partner (Pavitt, 2009), and a key factor informing those interpretations is the nature of both communicators. As Dautenhahn (2004) explains, when people interact with one another, they occupy the same ontological category (human), but when people interact with technology, each communicator belongs to a different category (human or machine). Researchers have found that people's conceptualizations of the nature of humans and machines matter for how they make sense of and interact with technology (e.g., A. P. Edwards, 2018; Sundar, 2008), but ongoing research is needed regarding how people conceptualize communicators occupying different ontological categories and the role of such conceptualizations in people's communicative behavior with technology (A. P. Edwards, 2018). This study continues this work by examining people's conceptualizations of the differences between humans and computers and how these differences inform people's perceptions of and interactions with communicative technologies.

The paper begins by explaining the study's focus on the nature of humans and computers before synthesizing foundational scholarship in artificial intelligence to identify existing ontological boundaries between people and computers. Next, HMC research regarding the nature of humans and machines is reviewed. This study was conducted by analyzing qualitative data regarding people's conceptualizations of the differences between humans and computers from two different projects regarding communicative technologies. The first part of the findings section presents the human-computer divides identified within the study, including new boundaries, while the second part explains the implications of these boundaries for human-machine communication. Finally, limitations and future research directions are presented.

\section{Literature Review}

\section{The Nature of Humans and Computers}

Human-Machine Communication as an area of research is so named to signal the difference in the nature of its communicators, with "machine" purposely used instead of "technology" as an acknowledgment of the long cultural, philosophical, and technological history regarding machines in relation to humans (see Guzman, 2018). In this study, the machine is conceptualized as a meta-ontological category encompassing multiple technologies. From antiquity, people have theorized what it is to be human in comparison and contrast to human-made items and nature (Boden, 2006; Riskin, 2007). As machines and society have evolved, so too have people's definitions of the boundary between themselves and machines (Turkle, 1984; Verbeek, 2005). The many permutations of the human-machine divide are 
too numerous and complex to examine here, and so this study focuses on the contemporary boundary between people and computers. The computer is positioned here as an ontological subcategory of the machine and encompasses the communicative technologies studied within HMC. Although many communicative technologies are accessed via hardware other than the computer, the computer is their technological progenitor.

From a historical and theoretical standpoint, the computer's introduction was a key turning point in the nature of machines (Turkle, 1984; Weizenbaum, 1976). In contrast to industrial machines that provide the "muscle" of manual labor, computers perform computations- “mental" processes once assumed to be unique to humans. Thus, computers challenge the ontological boundary between people and machines and, in doing so, the nature of each (Turkle, 1984).

Artificial intelligence research has further challenged the human-computer divide by using humans as prototypes for developing new technological capabilities (Boden, 2006; Dreyfus, 1999; Haugeland, 1985; Turkle, 1984). A key human capability being recreated within technology is the ability to communicate (Wachsmuth, 2008). The aim for imbuing technology with this ability is for interactions with devices to feel "natural" (Wachsmuth, 2008), similar to people's conversations with one another. The result has been technologies that are designed to exhibit human traits, such as gendered voices, and to emulate human communicative behaviors, such as using a person's name. The communicative technologies that are the subject of HMC research are the products of ongoing efforts to bridge the ontological gap between people and computers.

\section{Human-Computer Divides}

Ontologies are informed by people's worldviews (Chitty, 1997), and so the defining characteristics of the nature of humans and computers vary across individuals, cultures, and academic disciplines as do the boundaries between them. This review focuses on several key ontological divides between people and computers articulated regarding artificial intelligence, the study of which is focused on bridging these ontological boundaries (Franchi \& Güzeldere, 2005).

Origin of being. This boundary is predicated on the difference in how humans and computers come into existence. Machines have a single point of origin: they are made by humans (Franchi \& Güzeldere, 2005). People's perspectives on the genesis of humans, however, vary. A biological definition of humans situates people as part of the natural world and the product of evolution (Mazlish, 1993), while a spiritual perspective theorizes humans as divine beings created by a high power and superior to the rest of creation (Foerst, 2005) as the only beings to possess a soul (Minsky, 1986).

Autonomy. As an artificial object created by humans, the computer also is viewed as lacking another fundamental human trait-autonomy, the ability to act of one's volition (Boden, 2006; Suchman, 2011). Computers are programmed and, thus, viewed as being restricted to executing operations as directed by humans rather than having the free will of people. Relatedly, only humans are conceptualized as having a sense of self, an awareness of who they are in relation to the world around them (Haugeland, 1985; Mazlish, 1993). 
Emotion. The most straightforward articulation of this divide is that humans can feel and express emotion, computers cannot. It is this difference that has been key to how people perceive the ability of computers to make judgments in contrast to humans. Weizenbaum (1976) argues that within modernity, reason has been defined by the pursuit of science detached from feeling. Emotions, within this modernist perspective, are conceptualized as the antithesis of thought and reason (Minsky, 1986). As expressed by Bolter (1984), "the computer 'thinks' by means of dispassionate, logical, calculation" (p. 75) and, thus, is conceptualized as embodying the scientific ideal (Weizenbaum, 1976). For those who subscribe to this ideal, decisions rendered by computers are superior to those by humans whose emotions cloud their judgments.

However, it should be noted that such a view of the relationship between emotions and judgment has long been contested by some AI scholars (e.g., Minsky, 1986; Weizenbaum, 1976) and within more contemporary research within the social sciences (e.g., D. Evans, 2010; Vincent \& Fortunati, 2009). Emotion is a critical component of the communication process and how people interact with and make sense of their world, including the emotion people experience that is mediated by technology (Vincent \& Fortunati, 2009).

Intelligence. The introduction of the computer brought into question what it meant to have a "mind" and who, or what, could possess a mind (Turkle, 1984). The result was the prediction and establishment of parameters for "machine intelligence" (e.g., Turing, 1950) followed by an ongoing debate regarding how to achieve such intelligence, if doing so were even possible (e.g., Boden, 2006; Dreyfus, 1999; Minsky, 1986). Within these debates, the nature of intelligence itself has been contested (Boden, 2006; Kasabov, 2008). Today the question of whether a mind can be recreated within a machine remains unresolved (e.g., "Can We Copy the Brain?", 2017).

Communication. As Peters (2012) explains, philosophers such as Aristotle and Descartes viewed communication as a defining human trait. Beginning in the 19th century, the process of communication came to be culturally perceived as a critical element of the human experience (Peters, 2012). Within this context, the formal study of communication emerged and came to focus primarily on people (e.g., Schramm, 1982). In contrast, artificial intelligence and related fields have theorized communication as the exchange of information between sender and receiver, which can be a human or machine (see Guzman, 2018). The ontological divide within AI, and now communication following the establishment of HMC (Guzman \& Lewis, 2019), is not predicated upon whether people or computers communicate but on how they communicate. Within AI and related fields, research has focused on mitigating this divide between people and computers as communicators (e.g., National Aeronautics and Space Administration, 1976). A key approach in addressing this divide has been to replicate human traits within computers and to model human-computer interaction upon human communication (e.g., Licklider, 1960; Wachsmuth, 2008).

While the divides reviewed here are central within AI research, they alone cannot represent every facet of the human-computer divide. Ontological boundaries are shaped by the discipline in which they originate (J. H. Evans, 2016), and so, the preceding discussion based in the AI literature may not be representative of ontological perspectives in other fields. Recent research regarding cultural representations of technology also has found that 
elements of ontological definitions can vary between researchers and the public as well as overlap (e.g., Sarrica et al., 2019). Furthermore, ontologies can evolve, including in response to technology (Turkle, 1984).

\section{The Ontology of Communicators: Human and Computer}

When humans interact with other people, they rapidly take into account numerous aspects of their communication partner and this assessment, in turn, guides their communicative behavior (Pavitt, 2009). A key aspect of human communication that often is taken for granted is that this exchange is taking place between humans. This ontological sameness among communicators, Dautenhahn (2004) argues, allows for a series of assumptions to guide the interaction:

When faced with a human in a department store, we might ask ourselves, "Who is this?" . . Y Yet we know clearly what the person is-namely a member of the human species, which already allows us to make quite strong assumptions about his or her abilities, skills, and capacities. (p. 56)

But in human-machine communication, ontological assumptions may not be as straightforward. Dautenhahn (2004) continues, "In contrast, a robot or software agent in the role of a sales assistant leaves us widely in the open about its skills and capacities. Can it talk? Can it understand English? Does it know what the color blue is?" (p. 56). Interactions with machines, particularly initial interactions, require people to first determine the nature of the communicator, what it is, that then determines subsequent communication behavior (Dautenhahn, 2004). Exactly what people determine a digital interlocutor to be influences their assessment of it as a message source (Sundar \& Nass, 2001) and their judgment of how it should be treated (A. P. Edwards, 2018).

In human-human communication, people draw from their experience of communicating with humans and their personal knowledge of what it is to be a human to make sense of their communication partner. Within human-machine communication, the onus is on the human to decipher the nature of the communicative technology without the advantage of having shared experiences (National Aeronautics and Space Administration, 1976). As has been discussed, technologies designed as communicators also merge traits of people and computers and, in some instances, even animals. The question, then, is how people assign ontological categories to devices and programs that bring together traits across ontological boundaries (A. P. Edwards, 2018).

In a study of consumer judgments of news sources, Sundar and Nass (2001) introduce the "computer heuristic" to explain people's preference for a computer to select a news story for them because they perceive the computer's selection to be more "random" and, thus, less biased than a human's selection. Sundar (2008) relabels the "computer heuristic" as the "machine heuristic" and explains that this heuristic results in "attributions of randomness, objectivity, and other mechanical characteristics" to a computer's actions (p. 83). Because these characteristics are associated with unbiased judgment, people drawing upon this heuristic are likely to consider the computer a credible source (Sundar, 2008). What is not immediately clear, however, is what other traits people may associate with computers that also may influence their judgment of technology. 
A. P. Edwards (2018) observed that in interactions with a humanoid robot, some people acted toward the robot as they would another human, some people treated it similar to a pet, and others approached it as a computer. For A. P. Edwards, these differences hinted that people may be associating the robot with more familiar entities: humans, animals, and machines. Subsequent research by A. P. Edwards found that people think of animals and humans to be more similar to one another than a robot because they have a shared origin (biological or divine) separate from that of the human-built robot. These associations of ontological sameness and difference also had implications for how people judged other people's actions toward a robot.

Overall, people's interpretations of the nature of communicative technology is a crucial aspect of their judgment of and interactions with technology (Dautenhahn, 2004; A. P. Edwards, 2018; Sundar, 2008; Sundar \& Nass, 2001). Continued study of these interpretations is important to provide an understanding of how people perceive technology as a communicative other (A. P. Edwards, 2018) and begins with establishing how people conceptualize the nature of computers and humans. Ontological categories are defined by their boundaries (Franchi \& Güzeldere, 2005), and the study of the human-computer divide provides an understanding of the nature of each entity in addition to their differences (e.g., Turkle, 1984). AI research has identified key human-computer divides but given that ontological definitions can vary between scholars and the public (Sarrica et al., 2019), differ across academic fields (J. H. Evans, 2016), and evolve over time (Turkle, 1984), ongoing research is warranted. Therefore, the first research question focuses on how people differentiate between humans and computers. The second research question then focuses on the implications of these ontological divides for the process of human-machine communication.

\section{Method}

The data analyzed in this study was collected during two large-scale research projects regarding the ontology of disembodied artificial intelligence as communicator. The first project focused on voice-based, mobile AI assistants (e.g., Apple's Siri) (Guzman, 2015), while the second project, which began in 2018 and is ongoing, focuses on automated news-writing software (e.g., Automated Insights's Wordsmith). Both AI technologies can be considered disembodied because they are software without the type of physical form that research has shown to be key in people's interpretations of embodied communicators (e.g., Wachsmuth et al., 2008). Each project had specific research questions relative to the technology being studied, but the projects also had shared elements. Both projects focused on people's conceptualizations of a communicator that is of a different ontological nature than themselves and, therefore, examined how people conceptualize the ontological boundary between humans and computers. Bringing data from both projects into a single study enables more data points to be analyzed and for trends to be compared and contrasted regarding related technologies, adding to the robustness of the study's findings.

Both projects used the same protocol for sampling, data collection, and analysis. The method was semi-structured interviews because the goal of the research was to examine people's conceptualizations of novel technologies. Qualitative inquiry, generally, and interviews, in particular, are well-suited for research that is explanatory and exploratory and that 
foregrounds participants' knowledge (see Marshall \& Rossman, 1995). Following an inductive approach that enables participants to articulate their understanding of a technology also helps to fill in gaps between expert and user knowledge (Lee et al., 2014).

Purposive sampling was used in both projects. The researcher recruited participants in-person from public places (e.g., libraries, transit stations) within a large city in the United States' Midwest. Participants were selected based on factors germane to each project, including technology use (i.e., people using a certain technology or none at all), news consumption (automated news project), and demographic characteristics. Data from 46 of 64 participants within the mobile AI project and 27 of 29 people in the automated news project were analyzed for this study. The number of participants whose data is used in this study is fewer because some interviews did not discuss the nature of people and computers. The 73 participants included in this study ranged in age from 18 to $76(\mathrm{M}=37.7, \mathrm{SD}=$ 16.7): $59 \%$ identified as female, $40 \%$ as male, and $1 \%$ as non-binary. The racial and ethnic identity of participants was White (56\%), Black (16\%), Hispanic (11\%), Asian (10\%), Middle Eastern (3\%), mixed-race (3\%), and other (1\%). Highest level of education attained was graduate degree (16\%), some graduate school (11\%), bachelor's degree (40\%), associate or vocational degree (8\%), some college (18\%), or high school diploma (7\%). Participants primarily resided in urban (67\%) and suburban (23\%) areas. No trends were identified within the findings related to demographic characteristics, but findings should be viewed within the context of a sample of highly-educated participants living in an urban area.

Interviews were conducted in-person at the sites where participants were recruited. Participants selected an alias for the audio-recorded interviews and were assigned a new alias for this report. The interview protocol was developed around the concept of the "active interview" (Holstein \& Gubrium, 1997) that emphasizes the meaning-making process between researcher and participant. The questionnaire was developed from the literature and served as a guide for the interview with the course of each interview adapted to participant responses. This study focuses on participant answers to the question: "What is the difference between humans and computers?"

Data collection and analysis were concurrent, as is standard in qualitative research (Marshall \& Rossman, 1995). The endpoint of data collection was saturation-the point at which the data being collected becomes redundant and no longer contributes to new theoretical insight (Charmaz, 2014). Within the voice assistant project, saturation was based on the project's key research questions, not individual interview questions, but analysis of responses to the interview question asking people to differentiate between humans and computers showed distinct trends and redundancy in the data, indicating question-specific saturation. Aspects of the automated news project are ongoing, but the project has progressed enough to determine that saturation had been reached regarding people's conceptualizations of the differences between humans and computers.

Data analysis also was inductive. The researcher analyzed verbatim interview transcripts using MaxQDA software. To answer this study's first research question, the researcher analyzed responses regarding the difference between humans and computers. Analysis of the separate data sets began with in-vivo coding in which individual responses were coded with a descriptive phrase using a person's own words (see Saldaña, 2013). The researcher used the in-vivo codes to identify similarities within responses and then assigned new shared codes to grouped responses reflecting the same perspective. Data sets were merged, and the 
researcher continued this inductive coding process focused on identifying patterns within the data regarding aspects of human-computer divides. The researcher compared these emerging patterns to the AI literature to identify points of overlap and divergence before finalizing the divides discussed here. The study's second research question was answered by using participant responses as analyzed above to contextualize their opinions toward or interactions with disembodied AI programs as documented during other aspects of participant interviews.

\section{Findings}

\section{Conceptualizing the Human-Computer Divide}

Analysis resulted in several key findings that contribute to a greater understanding of the human-computer divide. Consistent with existing research (e.g., A. P. Edwards, 2018), people's conceptualizations of ontological boundaries are not uniform, but the differences articulated in this study are wider ranging. While mirroring ontological divides within the AI literature, participants identified additional boundaries based on the relationship between tools and tool-users and the flaws inherent to a particular group, most often humans. More than half of participants identified two or more differences between people and computers. Although scholars may categorize ontological divides as distinct, in the minds of some people, they are interrelated, with one divide often serving as the foundation for another. The percentage of participants identifying particular divides is included to provide a general sense of their prevalence but should be viewed within the limitations of this study's design. Interview quotes are edited for conciseness.

Origin of being. For $18 \%$ of participants, the origin of computers separated them from humans. Participants described the computer as an object that is "developed" or "programmed" by people. For example, Jeremy explains, "For the most part, I think that, of course, you know, computers are made by humans." Left unsaid in most of these responses is the origin of humans: It is enough that computers are made by people to be ontologically separate from them. Participants who do discuss people's origins reference their biological or divine nature. For example, Brad states: "So a computer, obviously, we know where a computer comes from. Humans, no idea. I mean, you can either believe god or you can trace your evidence, but nobody knows." Such answers are consistent with the AI and HMC literature (e.g., A. P. Edwards, 2018; Foerst, 2005).

The nature of the computer as constructed object also serves as the foundation for the other ways in which people differentiate between humans and computers. For Fatimah, because people build computers, they are "smarter." In contrast, Brenda thinks the artificial nature of the computer gives it an advantage over the biological limitations of people: "Humans make computers, so I kind of think, sometimes, computers may go farther than human beings because they never get tired." Conceptualizations of human and computer origins, thus, also shape people's perspectives of other traits inherent to each.

Tools and tool users. Closely related to the human-computer divide regarding origin of being is a divide focused on the nature of computers as "tools" and of people as tool users. This divide, which reflects a specific ontological relationship between people and 
computers, was expressed by $14 \%$ of participants. From this perspective, computers not only are made by humans but also are made to be used by humans. As Niel states: "Computers are machines developed by humans to serve humans and other functions." Niel's statement also captures another aspect of the difference between people and computers-that as machines, computers are intended to be of service to humans, a viewpoint also expressed by Joy: "I think computers are a tool to help us assemble information, disseminate information." Although the conceptualization of computers as tools and people as tool users is not identified within the AI literature discussed above, it is reflective of perspectives of the nature of people dating back more than a century (e.g., Carlyle, 1884) and of the nature of technology prominent within Western culture (e.g., Pacey, 1983).

Autonomy. The view of computer as a tool is one of many perspectives of technology that also feed into participant's conceptualizations of the differences in the levels of autonomy between computers and people. The ability to act of one's own volition is a divide between the nature of people and computers that has been discussed by AI scholars (e.g., Boden, 2006; Suchman, 2011) and, within this study, identified by $26 \%$ of participants.

Discussions of autonomy present people as possessing "free will" while the computer is restricted because it is programmed by humans, a reference back to its origins. Talula explains that ". . . humans, for the most part, we're autonomous in the way that we think. But machines are programmed to think a certain way or to arrive to a certain answer." This programmed nature results in a functional rigidity, according to participants like Dolores: "A computer has to be told every single thing to do. A computer can't think on its own like, 'Well, that didn't work, so let me try something else.' A computer has to be programmed." In contrast, people's ability to think autonomously provides them with mental flexibility not possessed by computers. Curtis further describes the difference: "I would say a computer is told what to do, and it does it. It's like a tool. I can feel one way about something, and then you could tell me, 'Hey, you should look at it this way', and then I might." Curtis's remarks also demonstrate the interplay among ontological boundaries: Because the computer is a programmed tool it cannot act on its own.

Intelligence. AI researchers have debated whether technology can possess human intelligence for more than 70 years (e.g., "Can We Copy the Brain?," 2017; Dreyfus, 1999), and today this question remains unresolved among scholars and, based on $44 \%$ of participants, the public as well. Participants identified multiple concepts related to intelligence, such as learning, knowledge, and decision-making, as points of delineation between people and computers. Some people defined this divide in terms of people possessing intelligence while computers do not. The majority of responses, however, reflected degrees of difference (Mazlish, 1993), with both people and computers possessing a trait but to varying degrees or as expressed differently.

Participants had wide-ranging perspectives on whether people or computers had the greater ability to acquire, process, and retain information. For Carol, humans possess greater intellect: "I think our brains are smarter than their's really." When asked to explain further, Carol adds:

We program computers. We've made them the holder, the place holder, of all of the things that we want to have at a moment's touch. But our brains have the 
capacity to be able to hang onto all kinds of infinite bits of information ... you think about how humanity started and the whole idea of 'how do you hold onto bits of information?'

Intellect, therefore, also is tied to the origin of humans and computers from Carol's perspective: That people have stored and passed along knowledge since the start of the species and then created computers to assist them elevates human intellect over that of the computer. Carol also uses the term "bit" - the vocabulary of the computer-in stating this difference, illustrating the argument by scholars that the way people understand and talk about the nature of humans and technology remains deeply intertwined (e.g., Agre, 1997; Mazlish, 1993).

Nicki is of a different opinion than Carol: "I feel like a computer would be more-I don't want to say smarter-but maybe more advanced than the average human." Explaining why, she says, "We can just say that maybe they [computers] have a bigger brain if you look at it that way. They're more knowledgeable." Nicki also would rather receive information from a computer because while people and computers are prone to "sources of error," the computer has less error.

The boundary between humans and computers intellectually also is built around the types of knowledge and information each possess and how they make sense of and act upon it. Flynn describes this difference in terms of acquired versus accumulated knowledge:

You have intellect. You have intelligence. You have acquired knowledge. You know, the AI is probably based on, as I know it, accumulations of knowledge. But we learn ... And that's one of the nice things about being a person is that as you live, you learn. I'm almost 70, and I still learn.

Both humans and computers have knowledge from Flynn's perspective, but what gives humans intelligence and sets them apart from computers is that their knowledge is the result of lived experience. In contrast, computers have compiled facts and figures but without this context.

For participants similar to Eleanor, the intellectual divide is more about the different strengths and weaknesses of computers and people than which one has superior intelligence:

Humans can think. Computers can compute. Computers can remember things. They retain information. They can find information. They do all kinds of stuff like that very quickly and very easily, and I certainly can't do that sort of thing. But a computer can't say, 'Does this make sense?'

Eleanor's answer points to a clear, but borderline tautological, divide between how people and computers deal with information, with computers excelling at some tasks and humans at others.

Emotion: Emotion also was a prominent divide, with $44 \%$ of participants saying the ability to feel and express emotion and to share in this process separates people from computers. Rachel explains that emotion is an exclusively human trait: "I mean the difference is definitely emotions . . . being able to feel compassionate for someone else, empathy, all that, 
sympathy." As with other divides, the line drawn over emotion can be shaped by people's views of the autonomy and origin of humans and computers. Janet explains that the computer lacks emotion because "ultimately it's already programmed by a human. It's still a machine. It's not designed as a real, thinking person would be." As an artificial object, computers cannot have emotion.

Aware of this ontological divide, developers have designed communicative technologies with emotional cues (e.g., Breazeal, 2002). Some participants who have interacted with these technologies consider emotion to be a shared trait between people and computers. During an interview regarding Siri, Rosa states “. . . it's kind of like bridging the line between technology and human emotions. I know you can tell it jokes, and it would respond to it sometimes ... ." Regarding what Rosa considers to be the difference between people and computers, she states, "Um, I don't know. Maybe it's just [the] sense where you can actually interpret the difference in sarcasm, humor. Because there's some things you can tell Siri, and it wouldn't register." For people like Rosa, technology can express emotion but not understand it.

For other participants, the fact that computers cannot understand emotion is central to the divide. Even when a communicative technology may seem to express emotion, participants like Brad argue that what is being conveyed is "perceived emotion." Andrew also explains further: ". . . you can’t really input empathy into technology that's genuine. Of course, you can have sort of false and pseudo-empathy." Similar to the nature of intelligence, which has become its own ontological debate within AI (Boden, 2006), the nature of emotion also is increasingly contested.

Emotions also continue to remain central to people's differing conceptualizations of judgments rendered by people and computers. For some participants, such as Amy, computers are better able to make certain types of decisions because they do not have emotions:

I think machines are able to make very hard decisions and are able to see everything through the constant lens of logic and rationality, whereas humans are very irrational and erratic, and there's change to them, and they're emotional and weird and kind of unpredictable, whereas machines are predictable and logical.

From Amy's perspective, emotions interfere with human judgment and emotionless technologies are better able to make certain decisions. However, other participants think the computer's lack of emotion is problematic for its judgment. Devon explains, "So it's kind of like the King Solomon thing, like split the baby in half. That's how computers would think, but we would think, 'Oh my god, that's terrible."' Devon is referencing a story from the Judeo-Christian tradition in which Solomon determines the rightful mother of a baby using a test of empathy. For Devon, who has a background in neuroscience, emotion and empathy provide people with a more complex understanding of the world that is vital to decision-making. Amy's remarks exemplify the cultural conceptualization of emotions as detrimental to judgment, while Devon's comments echo the counter-argument. Both signal that the debate over emotion, judgment, and technology has not abated among members of the public and may even become more complex if more people interpret technology as having emotion. 
Inherent flaws. Twelve percent of the responses from participants in this study offered a perspective of the difference between humans and computers that favored technology. These responses are separate from those previously discussed that weighed the advantages and disadvantages of human and computer traits tied to larger discussions of particular divides. The responses discussed here were offered as the primary reason humans and computers are different.

When asked to identify the difference between people and computers, Trisha replies, "I think that people make mistakes and do things inefficiently, and a computer will make less mistakes and do things more efficiently." Similarly, Natalie states, "I think humans are more flawed ... computers have their technical difficulties too, but in terms of the answers they'll give you, or the stuff they're supposed to do, humans could actually screw up a lot more in terms of that." Jaheim's initial response was to state that people do not have good intentions, adding that he has "met a few bad people" but should not "really use that as a judgment." Jaheim continues: "But I do think that computers could be more honest [than humans]. I mean pretty much if you tell a computer to do something, it will do that, and it will do that to the best of its ability." In contrast, Jaheim explains, humans "cut corners" and "try to slack."

The reason why some participants focused on the shortcomings of humans is not clear, and it is more difficult to locate these answers in ontological perspectives within the literature. However, the responses discussed here place the flaws of people, at least partly, within a task- or work-based context. Critical theorists of technology have argued that within a capitalist society, the development of performance standards around the capabilities of machines, such as efficiency, would result in people being viewed as inherently less than machines (e.g., Noble, 2011), an argument extended now to the ways in which people have to routinely verify their humanity (Fortunati et al., 2019). Such a judgment is outside the scope of this study but is a potential area for continued research.

Other divides. Human-computer divides representing less than $3 \%$ of responses include possessing a soul (human); consciousness (human); creativity (human); personality (human); mortality (human); mobility (human); and expendability (computers). Many of these divides appear in the data of only one project, potentially suggesting that certain boundaries may be associated with specific communicative technologies; however, the number of people identifying these divides is so small that it is not possible to reach a determination within this study.

\section{Implications for Human-Machine Communication}

The study's second research question focused on the implications of ontological boundaries for the human-machine communication process. The findings are drawn from participants' explanations of their judgments of or behaviors with communicative technology that were consistent with their answers to the interview question regarding the difference between humans and computers. As research has shown (e.g., Sundar, 2008; Sundar \& Nass, 2001), people's interpretations of the nature of a communicator play a role in their judgments of it, including within this study. Some participants' assessments of a technology's communicative abilities reflect how people differentiate between humans and computers. In addition, aspects of some people's communicative behavior with technology can be attributed to their 
interpretations of the human-computer divide, including whether they choose to interact with a technology and in what context.

One of the first decisions people make in human-machine communication is whether to even interact with a communicative technology. For some people, this decision is informed by their conceptualization of the boundary between humans and computers. Dolores differentiates between people and computers based on the computer's lack of autonomy that, in turn, negatively affects its decision-making. Dolores explains that "computers are not smart enough" to engage in verbal exchanges with people because they cannot process messages that deviate from their programming, and so she avoids interacting with Siri and similar technologies. Rachel also prefers communicating with people because, unlike computers, people possess and can understand emotion: "that's what I like with humans overall compared to computers is because they have feelings." And so, some people's conceptualizations of the human-computer divide play a role in determining whether they interact with a communicative technology at all.

For other people, decisions about communicating with technology hinge on the purpose of the interaction and whether a human or computer is better suited to achieving that goal. Nicki is an avid Siri user who thinks computers are "more knowledgeable" than people. When asked if she has ever turned to Siri for information instead of a human, she says, "Oh, yeah. Or even if I do [ask a person] I'll still use her [Siri] to verify." Nicki’s assessment of computers as possessing more information than people informs her decisions of when to interact with Siri instead of a person. Similarly, Curtis's perception of the human-computer divide over autonomy plays a role in his decision to interact with people and computers in different contexts:

When it comes to certain things, I'm gonna go with the computer. If it comes to how to do something, you know, how to feel, or if I'm looking at how to teach a lesson, I wanna listen to the teacher who's been doing it for 20 years over a computer program telling me, 'You should do this.' I think it's just that gray area that humans have that you can just go, 'Hmm, okay, I can take this and that'. A computer's gonna be just what it's told to give you.

Curtis weighs the intellectual autonomy of people against the programmed computer in choosing when to interact with one type of communicator instead of the other. Curtis's decision to turn to a human regarding his profession also may be indicative of how people consider not only ontological difference but also sameness in conceptualizing communicative others (e.g., A. P. Edwards, 2018).

The ontological boundary between humans and computers can also inform people's expectations of the capabilities and behaviors of technology during the human-machine communication process. For example, Tony thinks computers lack autonomy because they are programmed tools. Tony's conceptualization of the differences between humans and computers is reflected in how he contrasts the writing capabilities of human journalists with his expectations of news stories created by news-writing programs: "The difference between the two ... is that a human will bring in their own experiences, their own writing style, their own background... Whereas all the computer can do is take what's been programmed for it and bring it in." Tony perceives the software's communicative abilities will be limited because its autonomy is limited. 
As discussed, Rosa perceives Siri as "bridging" the human-computer divide regarding emotion and describes these interactions with Siri as "kind of weird." Rosa explains: “. . usually when you think about your phone, you think of technology. You think robotic. You think simple logistics. And so, when you start doing things like telling it jokes, it carries more human characteristics, I guess." What makes interactions with Siri "weird" is that Rosa is not expecting emotion to be part of a communicative exchange with a technology; this weirdness is not negative for Rosa who enjoys Siri. As A. Edwards et al. (2019) found, expectancy violations can take place in human-machine communication when the nature of the communicator or its behavior do not match a person's expectations for an interaction, as is the case in Rosa's interpretations of Siri.

People's conceptualizations of the human-computer divide play an important role in human-machine communication, including informing people's actions with or judgments of communicative technologies. As exemplified in Curtis's decision-making when selecting a communication partner, multiple factors, including ontological difference and sameness, inform people's decisions and actions in human-machine communication. Ontological boundaries, therefore, are important in people's communication with technology, but they are one of many elements shaping these interactions, as is the case with most aspects of communication generally.

\section{Conclusion}

The purpose of this study was to better understand people's conceptualizations of the ontological divide between humans and computers and the implications for human-machine communication. Many of the ontological boundaries within the foundational artificial intelligence literature - origin of being, autonomy, intelligence, and emotion-remain lines of delineation between people and computers from the perspective of the public. Some of these divides, however, are no longer as clear as they once were or are becoming even more complex. Most people consider emotion to be a key boundary, but some people's interactions with communicative technologies designed to emulate human emotions, such as Apple's Siri, have caused them to reassess the degree to which emotion remains a human trait. The debate regarding intelligence also continues; although, the question is not necessarily whether technology can be intelligent. For many people, both humans and computers have attributes of intelligence: Some people think humans are "smarter" while others think computers have bigger "brains." Relatedly, some people think aspects of human nature are inherently flawed when compared to that of computers, constituting a new divide. Based on these findings, scholars should be mindful that, although many of these divides are long-standing, they are not immune to change, particularly as technology design continues to integrate more human characteristics, such as emotion. The emergence of a new divide based on human flaws also underscores that these boundaries are not purely technological, they are social, and ongoing study is warranted to better understand the social aspects shaping these divides.

This study documents not only what people think divides humans and computers but also how people think about these divides. For most people, there is no singular ontological 
boundary; there are multiple divides, some of which serve as the foundation for others. Continued research is needed to better understand the relationship among these divides within people's minds, such as whether particular divides routinely serve as the basis for others, and to determine if certain boundaries are more influential than others in shaping people's interpretations of technology.

As the findings demonstrate, ontological boundaries have important implications for various aspects of the human-machine communication process. People's conceptualizations of the differences between humans and computers and the nature of each help to shape their overall interpretations of technology as a communicator. These interpretations then inform people's decisions and actions within the human-machine communication process. As documented here, perceived differences in the nature of humans and computers play a role in whether people choose to communicate with a particular technology, when people choose to communicate with a technology instead of a human, and what people expect from a technology as a communication partner. Because ontologies form the foundation of who or what something is as a communicator (Dautenhahn, 2004), it is likely that people's interactions with technology are influenced by ontological boundaries in other ways not identified here, and ongoing research is warranted to understand more fully how ontological divides between communicators shape interactions across these divides. Future research also will have to clarify the role of ontological divides as one of many factors that influence people's judgments of and actions with communicative technology.

Findings should be considered within the context of the study's limitations. The findings reported here are based on a sample of highly-educated people living in urban areas within the United States, and future research should take into account other populations or examine ontological boundaries across groups. The focus of the two research projects in which data was collected, disembodied AI technologies, also may have resulted in some boundaries being more pronounced, while others may be underrepresented or overlooked. For example, people's conceptualizations of the human-computer divide regarding mobility may be different in studies regarding embodied technologies, such as robots. Communication also was not identified as an ontological boundary, most likely because the focus of the research projects was on technologies that could carry out communicative functions. Ongoing research also is needed to determine which boundaries are universal among different types of technology and which are restricted to specific devices and programs. Finally, given the breadth of people's conceptualizations of the nature of humans and computers, HMC researchers should consider studying ontological aspects within the context of their own research. Adding questions or measures related to ontology may assist scholars in better interpreting their results.

\section{Acknowledgments}

This research was supported by a Research and Artistry Facilitation Grant from Northern Illinois University and a Faculty Research Grant from the Newspaper \& Online News Division of the Association for Education in Journalism and Mass Communication. 


\section{Author Biography}

Andrea L. Guzman ( $\mathrm{PhD}$, University of Illinois at Chicago) is an assistant professor of communication at Northern Illinois University (DeKalb, IL, USA) where she focuses on Human-Machine Communication research regarding people's perceptions of artificial intelligence technologies that function as communicators, including voice assistants and automated journalism software. Guzman's work has been published in leading journals, including New Media \& Society, Digital Journalism, and Computers in Human Behavior. Guzman is editor of Human-Machine Communication: Rethinking Communication, Technology, and Ourselves and the inaugural chair of the Human-Machine Communication Interest Group of the International Communication Association.

https://orcid.org/0000-0002-6874-9435

\section{References}

Agre, P. E. (1997). Computation and human experience. Cambridge University.

Boden, M. A. (2006). Mind as machine: A history of cognitive science (Vol. 1). Oxford University Press.

Bolter, J. D. (1984). Turing's man: Western culture in the computer age. University of North Carolina Press.

Breazeal, C. (2002). Designing socialable robots. The MIT Press.

Can We Copy the Brain? (2017, May 31). Archived at https://web.archive.org/web/ 20170622220350/http://spectrum.ieee.org/static/special-report-can-we-copy-the-brain

Carlyle, T. (1884). Sartor resartus: The life and opinions of Herr Teufelsdröckh. In Carlyle's Complete Works (The Vellum Ed). John Wilson \& Son, University Press.

Charmaz, K. (2014). Constructing grounded theory (2nd ed.). SAGE.

Chitty, A. (1997). First person plural ontology and praxis. Proceedings of the Aristotelian Society, 97, 81-96.

Dautenhahn, K. (2004). Socially intelligent agents in human primate culture. In S. Payr \& R. Trappl (Eds.), Agent culture: Human-agent interaction in a multicultural world (pp. 45-71). https://doi.org/10.1201/b12476

Dreyfus, H. L. (1999). What computers still can't do: A critique of artificial reason (6th ed.). MIT Press.

Edwards, A., Edwards, C., Westerman, D., \& Spence, P. R. (2019). Initial expectations, interactions, and beyond with social robots. Computers in Human Behavior, 90, 308-314. https://doi.org/10.1016/j.chb.2018.08.042

Edwards, A. P. (2018). Animals, humans, and machines: Interactive implications of ontological classification. In A. L. Guzman (Ed.), Human-Machine Communication: Rethinking communication, technology, and ourselves (pp. 29-50). https://doi.org/10.3726/b14399

Evans, D. (2010). Emotion: A very short introduction. Oxford University Press.

Evans, J. H. (2016). What is a human? What the answers mean for human rights. Oxford University Press. 
Foerst, A. (2005). Artificial intelligence and theology: From mythos to logos and back. In S. Franchi \& G. Güzeldere (Eds.), Mechanical bodies, computational minds: Artificial intelligence from automata to cyborgs (pp. 489-513). MIT Press.

Fortunati, L., Manganelli, A. M., Cavallo, F., \& Honsell, F. (2019). You need to show that you are not a robot. New Media \& Society, 1-18. https://doi.org/10.1177/1461444819831971

Franchi, S., \& Güzeldere, G. (Eds.). (2005). Machinations of the mind. In Mechanical bodies, computational minds: Artificial intelligence from automata to cyborgs (pp. 15-151). MIT Press.

Guzman, A. L. (2015). Imagining the Voice in the Machine: The Ontology of Digital Social Agents. University of Illinois at Chicago, Chicago, IL.

Guzman, A. L. (2018). What is human-machine communication, anyway? In A. L. Guzman (Ed.), Human-Machine Communication: Rethinking communication, technology, and ourselves (pp. 1-28). Peter Lang. https://doi.org/10.3726/b14399

Guzman, A. L., \& Lewis, S. C. (2019). Artificial intelligence and communication: A Human-Machine Communication research agenda. New Media \& Society. https://doi. org/10.1177/1461444819858691

Haugeland, J. (1985). Artificial intelligence: The very idea. The MIT Press.

Holstein, J. A., \& Gubrium, J. F. (1997). Active interviewing. In D. Silverman (Ed.), Qualitative research: Theory, method, and practice (pp. 113-129). SAGE.

Kasabov, N. (2008). Evolving intelligence in humans and machines: Integrative evolving connectionist systems approach. IEEE Computational Intelligence Magazine, 3(3), 23-37. https://doi.org/10.1109/MCl.2008.926584

Lee, H. R., Šabanovic, S., \& Stolterman, E. (2014). Stay on the boundary: Artifact analysis exploring researcher and user framing of robot design. Presented at the ACM-CHI (pp. 1471-1474). ACM Press. https://doi.org/10.1145/2556288.2557395

Licklider, J. C. R. (1960/1990). Man-computer symbiosis. In R. W. Taylor (Ed.), In memoriam: J. C. R. Licklider 1915-1990 (pp. 1-20). Systems Research Center.

Marshall, C., \& Rossman, G. B. (1995). Designing qualitative research (2nd ed.). SAGE.

Mazlish, B. (1993). The fourth discontinuity. Yale University Press.

Minsky, M. L. (1986). The society of mind. Simon \& Schuster.

National Aeronautics and Space Administration. (1976). A forecast of space technology 1980-2000 (No. NASA SP-387). Washington, DC.

Noble, D. F. (2011). Forces of production: A social history of industrial automation. Transaction Publishers.

Pacey, A. (1983). The culture of technology (First MIT Press). MIT Press.

Pavitt, C. (2009). Impression formation. In S. W. Littlejohn \& K. A. Foss (Eds.), Encyclopedia of communication theory (pp. 203-206). SAGE.

Peters, J. D. (2012). Speaking into the air: A history of the idea of communication. University of Chicago Press.

Riskin, J. (Ed.). (2007). Genesis redux: Essays in the history and philosophy of artificial life. University of Chicago Press.

Saldaña, J. (2013). The coding manual for qualitative researchers (2nd ed.). SAGE.

Sarrica, M., Brondi, S., \& Fortunati, L. (2019). How many facets does a "social robot" have? A review of scientific and popular definitions online. Information Technology \& People. https://doi.org/10.1108/ITP-04-2018-0203 
Schramm, W. (1982). Men, women, messages, and media: Understanding human communication (2nd ed.). Harper \& Row.

Suchman, L. (2011). Subject objects. Feminist Theory, 12, 119-145. https://doi.org/ $10.1177 / 1464700111404205$

Sundar, S. S. (2008). The MAIN model: A heuristic approach to understanding technology effects on credibility. In M. J. Metzger \& A. J. Flanagin (Eds.), Digital media, youth, and credibility (pp. 73-100). Cambridge, MA: The MIT Press.

Sundar, S. S., \& Nass, C. (2001). Conceptualizing sources in online news. Journal of Communication, 51, 52-72. https://doi.org/10.1111/j.1460-2466.2001.tb02872.x

Turing, A. M. (1950). Computing machinery and intelligence. In D. C. Ince (Ed.), Mechanical Intelligence (pp. 133-160). North-Holland.

Turkle, S. (1984). The second self. Simon \& Schuster.

Verbeek, P. P. (2005). What things do: Philosophical reflections on technology, agency, and design (English Translation). Pennsylvania State University Press.

Vincent., J., \& Fortunati, L. (2009). Electronic emotion: The mediation of emotion via information and communication technologies. Peter Lang.

Wachsmuth, I. (2008). 'I, Max'-Communicating with an artificial agent. In I. Wachsmuth \& G. Knoblich (Eds.), Modeling Communication with Robots and Virtual Humans (Vol. 4930, pp. 279-295). https://doi.org/10.1007/978-3-540-79037-2_15

Wachsmuth, I., Lenzen, M., \& Knoblich, G. (Eds.). (2008). Embodied communication in humans and machines. Oxford University Press.

Weizenbaum, J. (1976). Computer power and human reason: From judgment to calculation. W.H. Freeman \& Co. 